\title{
Thermal transport in glassy selenium: The role of low-frequency librations
}

\author{
F. J. Bermejo \\ Instituto de Estructura de la Materia, Consejo Superior de Investigaciones Cientificas, Serrano 123, E-28006 Madrid, Spain \\ E. Enciso \\ Departamento de Química-Física I, Universidad Complutense de Madrid, Madrid E-28040 Spain \\ A. Criado \\ Departamento de Física de la Materia Condensada, Instituto de Ciencia de Materiales, Universidad de Sevilla, \\ P.O. Box 1065, E-41080 Sevilla, Spain \\ J. L. Martínez \\ Instituto de Ciencia de Materiales, Departamento de Física-Aplicada C-IV, Facultad de Ciencias, \\ Universidad Autónoma de Madrid, Madrid E-28049, Spain \\ M. Garcia-Hernández \\ Instituto de Estructura de la Materia, Consejo Superior de Investigaciones Cientificas, Serrano 123, E-28006 Madrid, Spain
}

(Received 3 August 1993; revised manuscript received 7 October 1993)

\begin{abstract}
The experimental curves giving the temperature dependence of the thermal conductivity of glassy selenium are considered in detail. The observed behavior can be taken into account quantitatively if the densities of states for short-wavelength phonons as well as for low-energy librations arising from computer simulations are used for the calculations. In particular, it is shown that the lowestfrequency excitations of a chain of selenium atoms can give due account of the plateau observed at temperatures about 2-10 K. The implications of the present findings for the current debate regarding the mechanisms for thermal transport in glasses are finally discussed.
\end{abstract}

\section{INTRODUCTION}

Several significant developments towards the understanding of the thermal anomalies characteristic of the glassy state have appeared in recent times. ${ }^{1}$ Within those, two widely different approaches, either couched in purely phenomenological (i.e., Ref. 2) or semimicroscopic ${ }^{3,4}$ terms, have evidenced a similar predictive capability, albeit involving rather different physics. The first family of approaches, which is close in spirit to the celebrated two-level-system (TLS) model, ${ }^{5}$ starts from consideration of some anharmonic (quartic) potential without making reference to the microscopic entities executing such anharmonic motions, and by means of bilinear coupling of the modes corresponding to such potentials to the strain fields is able to account for those features characterizing the glassy dynamics, whereas the second type of approach makes use of microscopic concepts ("elastic dipoles" or other stress sources",3) which couple to phonons by means of several mechanisms. In such cases, specific microscopic entities may be found as responsible for the observed anomalies, thus providing a firmer understanding of the underlying mechanisms driving the glassy dynamics.

This contribution presents a quantitative interpretation of the thermal conductivity of an archetypal glass: selenium, which has recently been analyzed on phenomenological grounds. ${ }^{2}$ We will make use of some microscopic information available from computer molec- ular dynamics (MD) simulations such as several frequency distributions characterizing different atomic motions. Contrary to other approaches where this transport property is directly calculated from the pertinent autocorrelations, ${ }^{6}$ our purpose here is to identify which microscopic entities originate the glassy anomalies in this, topologically disordered solid. In this respect, it is worth noting that only in cases where some well-defined structural units exist (molecular dipoles in the case of mixed cyanides, ${ }^{4}$ or concerted motions of $\mathrm{SiO}_{4}$ tetrahedra in vitreous silica ${ }^{7}$ ) has such an approach been carried out. The present work follows previous accounts regarding neutron scattering and computer simulation data ${ }^{8}$ where, in common with some other materials,${ }^{9}$ the temperature dependence of the specific heat at intermediate temperatures was accounted in semiquantitative terms if the $Z(\omega)$ densities of states obtained from simulation means were used for the calculation. The thermal conductivity of a crystalline sample is also analyzed as a reference, using for the purpose the density of states calculated from a previous lattice dynamics calculation. ${ }^{8}$

The next section gives details of the calculations and numerical values for the employed model parameters. At low temperatures the simulation data have to be supplemented with some additional framework in order to account for very low frequencies and long wavelengths. Because of its widespread use, recourse was made to the standard TLS phenomenology to cover this energy region ( $\approx$ below $2 \mathrm{~K}$ ), although some other alternatives could also be plausible. 


\section{DETAILS OF THE CALCULATIONS}

\section{A. Basic formulas and relevant parameters}

We start, as customarily done, by expressing the thermal conductivity by means of the kinetic theory result,

$$
\begin{aligned}
\kappa(T) & =\int_{0}^{\omega_{\max }} d \omega C_{v}(\omega, T) \bar{v}(T) l(\omega, T) \\
C_{v}(\omega, T) & =\int_{0}^{\omega_{\max }} d \omega\left[Z_{\mathrm{c} . \mathrm{m} .}(\omega)+\bar{P}_{\mathrm{TLS}}\right] \frac{x^{2} e^{x}}{\left(e^{x}-1\right)^{2}}
\end{aligned}
$$

where $\omega_{\max }$ is the maximum frequency up to which the $Z_{\text {c.m. }}(\omega)$ density of states of the glass as projected onto the molecular center of mass (c.m.) of the selenium chains located at $R_{\text {c.m. }}(t)$ positions with $v_{\text {c.m. }}(t)$ velocities, i.e.,

$$
Z_{\text {c.m. }}(\omega)=\frac{1}{\pi} \operatorname{Re} \int_{0}^{\infty} d t \exp (-i \omega t)\left\langle v_{\text {c.m. }}(t) \cdot v_{\text {c.m. }}(0)\right\rangle
$$

shows appreciable values. The choice of such a quantity instead of the total atomic $Z(\omega)$ is based upon the fact ${ }^{10}$ that, from analysis of the several components of the latter quantity, the $Z_{\text {c.m. }}(\omega)$ exhibits harmonic behavior in the temperature range of interest, and on the other hand, predicts a temperature dependence of the specific heat in an acceptable agreement with experiment. ${ }^{8}$ The specific heat of the phonons of frequency $\omega$ and temperature $T$ is given by $C_{v}(\omega, T)$ with $x=\hbar \omega / k_{B} T$, and $\bar{P}_{\mathrm{TLS}}$ accounts for the contribution from two-level systems which is supposed to become dominant below $1 \mathrm{~K}$, the value of which is usually obtained from fits to low-temperature specific heat data. The average phonon velocity $\bar{v}(T)$ is assumed to be frequency independent, and its temperature dependence was taken from experimental ultrasound work. ${ }^{11}$ The last quantity entering Eq. (1) $l(\omega, T)$ is the frequency- and temperature-dependent phonon mean free path, an adequate modelization of which is required to explain the observed anomalies in thermal transport. Since a number of phonon scattering mechanisms are known to operate in glasses, the resulting scattering rate is obtained making use of the Matthiesen recipe of adding independent scattering rates, $l^{-1}(\omega, T)=l_{\mathrm{TLS}}^{-1}(\omega, T)+$ $l_{\text {relax }}^{-1}(\omega, T)+l_{\text {Rayl }}^{-1}(\omega, T)+l_{\text {libr }}^{-1}(\omega, T)$. The first contribution stands for the dominant phonon-scattering mechanism at low temperatures by means of resonant scattering from the two-level systems, and is included in order to complement the simulation with those effects not easily amenable to computational means. For its modelization, we use the standard expression ${ }^{5}$

$$
l_{\mathrm{TLS}}^{-1}(\omega, T)=A(T) \omega \tanh \left(\frac{\hbar \omega}{2 k_{B} T}\right),
$$

where the $A$ coefficient is given in terms of the strain coupling of the TLS to phonons, $\bar{P}_{\text {TLS }}$, the density of TLS states, and material constants (average velocity of sound and density). From values of the coupling constant and the density of TLS taken from Ref. 2, an estimate for this coefficient gives a value for $T=1 \mathrm{~K}$ of $A=5.861 \times$
$10^{-4} / \bar{v}(T=1 \mathrm{~K})$. The second contribution arises from relaxational absorption due to the phonon modulation of the level splitting of the TLS which leads to a phonon scattering rate $^{12}$

$$
l_{\text {relax }}^{-1}(\omega, T)=\frac{1}{\bar{v}(T)}\left[\frac{1}{A_{\text {relax }} T^{3}}+\frac{1}{B \omega}\right]^{-1},
$$

where the strength factor $A_{\text {relax }}$ can again be computed from values of the TLS constants and material parameters and $B=A / 2$. The value calculated for this parameter at the same reference temperature than above is thus $A_{\text {relax }}=1.7951 \times 10^{5} \mathrm{~s}^{-1} \mathrm{~K}^{-1}$. An additional mechanism for phonon scattering in glasses, the importance of which has been the subject of extensive discussion ${ }^{4}$ regards Rayleigh scattering by fluctuations of the elastic constants. An estimate of this contribution is given by ${ }^{13}$

$$
\begin{aligned}
l_{\text {Rayl }}^{-1}(\omega, T) & =\frac{1}{\bar{v}(T)}\left[\tau_{\min }+\frac{1}{R(T) \omega^{4}}\right]^{-1}, \\
\tau_{\min } & \approx \frac{r_{0}}{\bar{v}(T)}, \quad R(T)=\frac{r_{0}^{3} F}{4 \pi \bar{v}^{3}(T)}, \\
F & =\sum_{i} f_{i}\left(\Delta M_{i} / \bar{M}\right)^{2} .
\end{aligned}
$$

Here, $\tau_{\min }$ represents a cutoff for short wavelengths which ensures that the mean free path cannot become shorter than the characteristic scale for density fluctuations $r_{0}$. For this latter parameter the value of 7.85 $\AA$ was chosen, since it corresponds to the wave vector where a small prepeak was found in the strictly elastic $S(Q, \omega=0)$ dynamic structure factor measured in a recent experiment. ${ }^{10}$ Finally, the value of $F$ giving a weighted average deviation from the average mass $\bar{M}$ was computed following the same procedure employed in the pioneering work of Zeller and Pohl. ${ }^{14}$ The value for the Rayleigh coefficient so computed as well as for the cutoff was, again for $T=1 \mathrm{~K}, R=4.82 \times 10^{-40}$ $\mathrm{s}^{-3}$, and $\tau_{\min }=0.39 \times 10^{-12} \mathrm{~s}$. As it will be shown below, and seems to be common to a number of glasses either orientational or topologically disordered, ${ }^{4}$ an additional scattering mechanism needs to be added since otherwise the calculated thermal conductivity will be far in excess of the experimental values in the plateau region. For such a purpose, the density of states $Z_{\text {libr }}(\omega)$ for low-amplitude motions, which in the case of selenium corresponds to torsional librations of the chain along the Se-Se bonds was computed from the MD simulation. The first peak of such a frequency distribution as shown in Fig. 1 is located at about $1.2 \mathrm{THz}$, and corresponds to pure torsional oscillations whereas higher-frequency features peaking at $2.8 \mathrm{THz}$ and $\approx 7 \mathrm{THz}$ arise from the coupling of these motions to the other internal degrees of freedom of the Se chains (bond-angle bending and bond stretching). The maximum at $1.2 \mathrm{THz}$ is, on the other hand, reasonably close to the value of $1.51 \mathrm{THz}$ calculated from the barrier height $V_{0}=16.66 \mathrm{THz}$ (Ref. 8) and the moment of inertia of a group of four Se atoms which gives $I_{r}=35.34 \mathrm{~g} \mathrm{~cm}^{2}$ as $\omega_{t}=\sqrt{\left(V_{0} / 2 I_{r}\right)}$. The same relationship could be used to derive the effective moment of inertia (assuming the barrier height to be equal) from the MD frequency distribution, which gives 
$I_{\text {eff }}=55.63 \mathrm{~g} \mathrm{~cm}^{2}$. For the calculation of the scattering rate due to low-energy librations, we have followed a similar procedure to that employed for the analysis of $\mathrm{KBr}: \mathrm{KCN}$ orientational glasses, ${ }^{4}$ thus getting

$$
\begin{aligned}
l_{\mathrm{libr}}^{-1}(\omega, T) & =\frac{1}{\bar{v}(T)} \int d \omega \prime Z_{\mathrm{libr}}(\omega) \chi_{t}\left(\omega-\omega \prime, \omega_{t}\right) \\
\chi_{t}\left(\omega, \omega_{t}\right) & =4 \pi \bar{v}^{3}(T)[n(\omega)+1] \frac{4 \omega^{2} \omega_{t} \Gamma(\omega)}{\left(\omega^{2}-\omega_{t}^{2}\right)^{2}+4 \omega^{2} \Gamma^{2}(\omega)},
\end{aligned}
$$

where $n(\omega)$ is a Bose factor, and the scattering rate is given as a convolution of the density of states for low-frequency librations with the line shape of harmonic phonons which are scattered off libration modes of frequency $\omega_{t}$. This introduces a frequency-dependent damping which is given by ${ }^{4}$

$$
\Gamma(\omega)=\frac{\hbar \omega^{2} \Lambda_{c}^{2}}{4 \pi \rho I_{\mathrm{eff}} \bar{v}^{5}(T)}
$$

in terms of material parameters (density $\rho$ assumed temperature independent, and average sound velocity) and $\Lambda_{c}$, the coupling constant of the librators to the strain field, for which a value of $0.5 \mathrm{eV}$ was taken following data quoted in Refs. 2 and 4.

As it will be shown below, the wide variations in $\kappa(T)$ observed upon different thermal treatments imply that some adjustment of some of the parameters has to be performed to reproduce the experimental shapes. As a consequence the normalization constant for $Z_{\text {libr }}(\omega)$, the $\bar{P}_{\text {TLS }}$ constant density of TLS states and a factor correcting for variations in the velocity of sound were used as the only three adjustable parameters for the explored temperature range.

A somewhat different procedure was employed for the analysis of the crystal data. The relevant density of states entering Eq. (2) is now the $Z_{\text {cryst }}(\omega)$ frequency distribution calculated by means of a lattice dynamics procedure, ${ }^{8}$ and the number of terms entering the inverse mean free path is now reduced to $l_{\text {cryst }}^{-1}(\omega, T)=$ $l_{\mathrm{bs}}^{-1}+l_{3 \mathrm{ph}}^{-1}(\omega, T)+l_{\text {Rayl }}^{-1}(\omega, T)$, where the first term accounts for boundary scattering effects, the anharmonic contributions are lumped together in a three-phonon term, which is supposed to be the leading contribution for the temperature range of interest, and the Rayleigh term now includes isotopic difference scattering as well as some other sources having the same frequency dependence (for a detailed discussion of all these topics see Ref. 13). The explicit form for these contributions are given by ${ }^{15}$

$$
\begin{aligned}
& l_{\mathrm{bs}}^{-1}=1 /(G L), \quad l_{3 \mathbf{p h}}^{-1}(\omega, T)=B \omega^{2} T^{3} / \bar{v}(T), \\
& l_{\text {Rayl }}^{-1}(\omega, T)=A_{\text {Rayl }} \omega^{4} / \bar{v}(T) .
\end{aligned}
$$

Here, $G$ stands for a parameter normally needed to account for boundary scattering of phonons at lengths somewhat different than the Casimir length $L$, and $B$ is a parameter giving account of phonon decay processes. Finally, the value of $A_{\text {Rayl }}$ also has to be optimized since, as evidenced from the analysis of a considerable number of insulators and semiconductors, ${ }^{13}$ it usually exceeds the value calculated from the mass-difference ratios. No contribution of scattering by donors or acceptor holes seem to be needed for the case of glassy or crystalline Se. The conductivity of the crystalline sample was thus analyzed using $G, B$, and $A_{\text {Rayl }}$ as three adjustable constants.
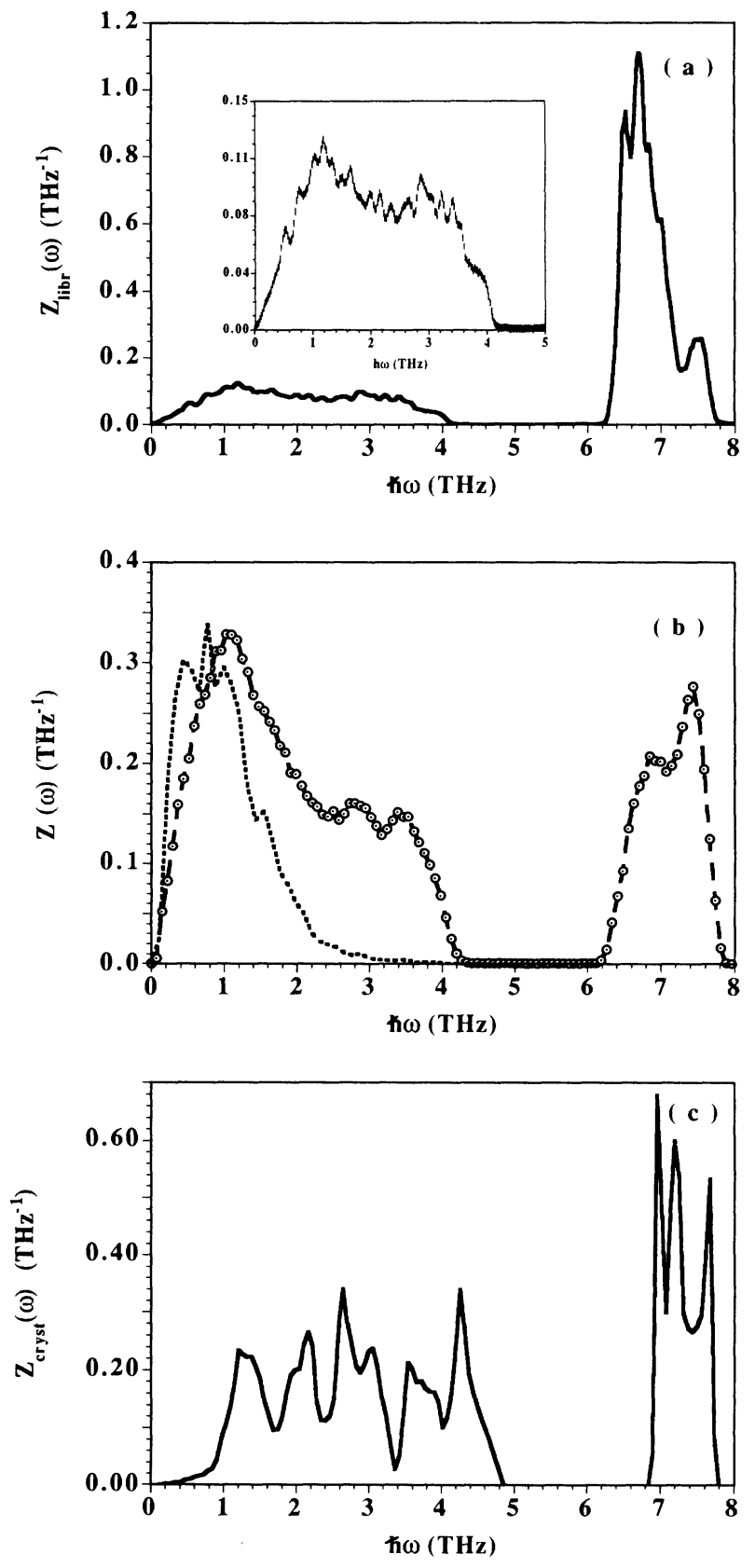

FIG. 1. (a) Density of states of low-frequency librations $Z_{\text {libr }}(\omega)$ as calculated from the MD computations (see text). The inset shows a blow up of the calculated function for the frequency extent relevant to intermediate temperatures. The curve has been normalized here to $1 \mathrm{THz}$. (b) The total density of vibrational states for $T=15 \mathrm{~K}$ (circles with a dot) and the projection onto the c.m., $Z_{\text {c.m. }}(\omega)$. The total function is normalized to $1 \mathrm{THz}$ and the c.m. counterpart has been scaled according to coefficients given in (9) (see text). (c) The total density of states for a crystalline sample as calculated from the harmonic lattice dynamics computations. 


\section{B. Computer simulation}

The details concerning the validation of the model potential employed for the simulation, system characteristics, and overall performance of the calculated quantities have already been given. ${ }^{8}$ Additional calculations were carried out for low-temperature samples $(15 \mathrm{~K})$ as well as to study with greater detail the influence of the chain length on the dynamics and transport properties of the melts. ${ }^{16}$ The calculation of $Z_{\text {libr }}(\omega)$ was performed from the autocorrelation functions corresponding to torsionangle movements, and a detailed discussion of the internal dynamics of the Se chains in the glassy state, along the lines employed for the study of the melt ${ }^{16}$ will be discussed elsewhere. The scaling of the $Z_{\text {c.m. }}(\omega)$ contribution to the total atomic density of states was achieved following the procedure already described which is based on the analysis of the fluctuations of the configurational energy corresponding to the different contributions to the interaction potential (for explicit values see Table I of Ref. 8). From the simulation length and system size characteristics, reliable estimates of the relevant autocorrelations extend down to $\approx 0.05 \mathrm{THz}(\approx 2.4 \mathrm{~K})$ and $\approx 27.5 \AA$. In order to extend the frequency range to cover the low-temperature region, the $Z_{\text {c.m. }}(\omega)$ were extrapolated at zero frequencies using a Debye law which joined smoothly the simulated data, using for the purpose average sound velocities modified to fit the experimental data as it is discussed below.

\section{RESULTS}

The calculated frequency distributions for all the excitations at $T=15 \mathrm{~K}$, its projection into the c.m. of the Se chains, the librational density of states for the same temperature, as well as the crystalline $Z_{\text {cryst }}(\omega)$ function are displayed in Fig. 1. Only some comments regarding $Z_{\text {libr }}(\omega)$ are required here, since the relevant discussions and comparison between the densities of states of crystal and glassy samples have already been given. ${ }^{8,10}$ The three broad features apparent in this function indicate that, as expected, the torsional librations are strongly coupled to the other two internal degrees of freedom of the Se chains. Within the temperature range of interest, only the first two peaks of this latter distribution are significant, and the shape of the distribution up to $5 \mathrm{THz}$ can be approximated reasonably well by a sum of two Gaussians with mean and variances of $1.15 \mathrm{THz}(55 \mathrm{~K})$, $0.5 \mathrm{THz}$ and $2.87 \mathrm{THz}(137.7 \mathrm{~K}), 0.68 \mathrm{THz}$, respectively.

The intermediate temperature $(3-300 \mathrm{~K})$ data for glassy and crystalline Se were taken from Meissner et $a l .{ }^{17}$ and two sets of low-temperature data $(0.1-10 \mathrm{~K})$ for ' a quenched glass ${ }^{14}$ and for a quenched glass and a sample annealed at $278 \mathrm{~K}$ for 30 days under irradiation by a high-flux $\gamma$ beam (1.2 Mrad/h) (Ref. 18) were also considered. Figure 2(a) shows the experimental data for intermediate temperatures for a rapidly quenched glass, a sample which was annealed at room temperature for 6 months, as well as a sample annealed at $303 \mathrm{~K}$ for $2 \mathrm{~h}$. It can be seen that the approximation followed herein is able to reproduce the experimental data up to $200 \mathrm{~K}$ with acceptable accuracy for both the quenched glass and the sample annealed at room temperature. The difference in both curves can be accounted by a noticeable variation of the constant normalizing the librational density of states which goes from a value of $0.82 \times 10^{22} \mathrm{~cm}^{-3}$ in the first case to $0.53 \times 10^{22} \mathrm{~cm}^{-3}$ in the latter. Also, the parameter $\bar{P}_{\text {TLS }}$ specifying the frequency-independent TLS density
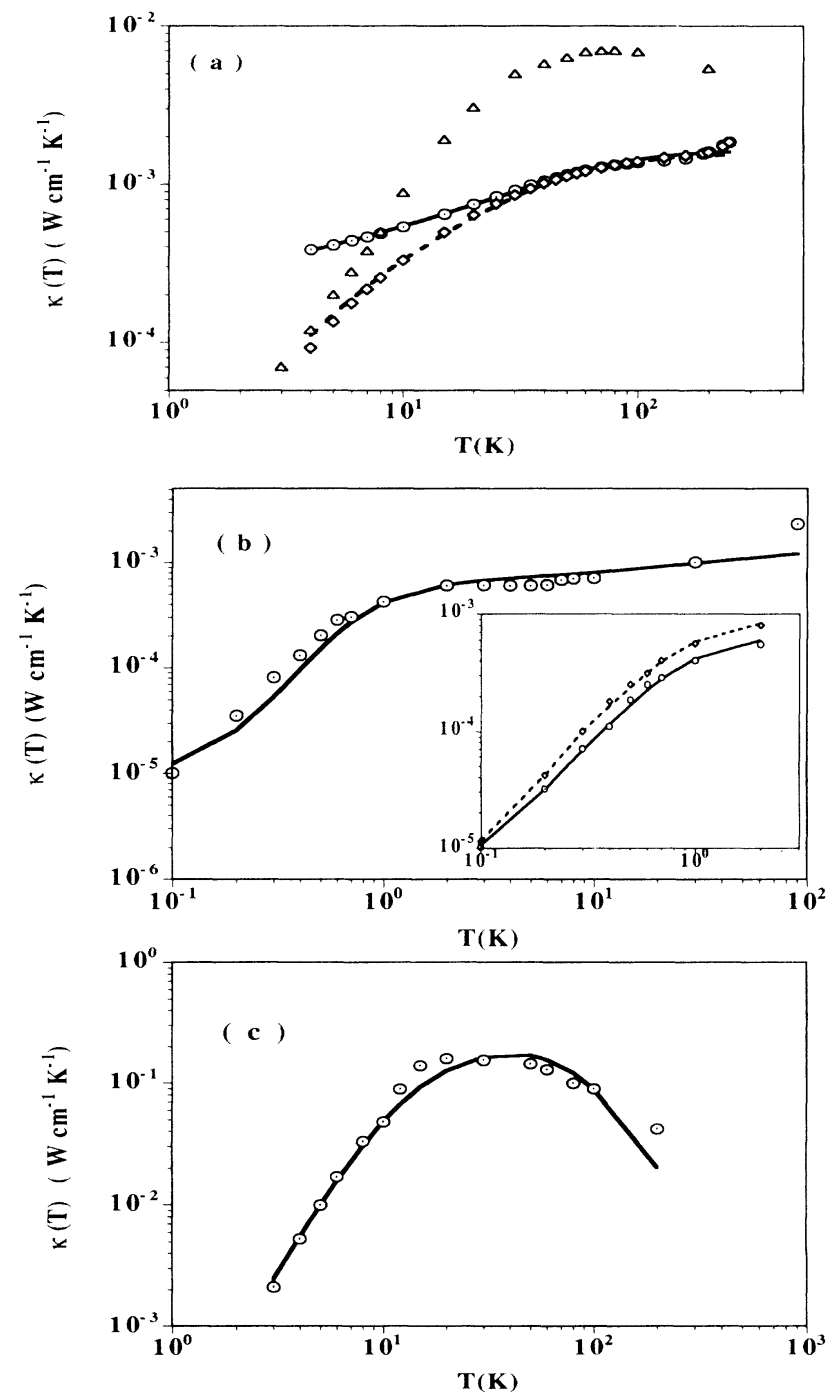

FIG. 2. (a) Thermal conductivities at intermediate temperatures. Open circles show the experimental data of Meissner et al. (Ref. 17) for a rapidly quenched glass and the solid line shows the present approximation to the data. The open lozenges and the dashed line show the experimental and calculated result for a glass annealed at room temperature for 6 months. The open triangles show the measured data for a sample annealed at $303 \mathrm{~K}$ for $2 \mathrm{~h}$ (see text). (b) The open circles and the solid line show the experimental data (Ref. 15) and the approximation discussed in the text. The inset shows the data of Ref. 18 for a quenched glass (circles and solid line) and an annealed-under-irradiation sample (lozenges and dashed line). (c) The conductivity for a polycrystalline solid as measured by Meissner (Ref. 17) (circles) as well as its approximation as described in the text (solid line). 
of states goes from a value of $4.6 \times 10^{39} \mathrm{~J}^{-1} \mathrm{~cm}^{-3}$ for the quenched glass to $1.4 \times 10^{37} \mathrm{~J}^{-1} \mathrm{~cm}^{-3}$ in the second, and concurrently, the Debye velocity of sound experiences an increase of up to $7 \%$ upon annealing. The curves corresponding to the sample annealed at a higher temperature, shown in Fig. 2(a) for comparison purposes, could not be reproduced acceptably (exception made of the lowtemperature portion), and the high conductivity about $50 \mathrm{~K}$ suggests that, as it will be shown below, some partial crystallization may have occurred.

From $4 \mathrm{~K}$ onwards, the scattering processes arising from TLS do not seem to have any measurable influence and, as a matter of fact the curves could be also reproduced ignoring the two-level terms. It is then clear that at a temperatures characteristic of the first plateau and above, torsional librations about the Se-Se bonds are the main mechanism limiting heat transport. The highertemperature data (above $200 \mathrm{~K}$ ) deviate systematically from our model, something which may well indicate the need of including thermal expansion and anharmonic effects, a topic seldom considered in the literature regarding glasses but evidenced as increasingly important for temperatures above ca. $10 \mathrm{~K}$ in crystalline materials. ${ }^{13}$

The low-temperature data $^{14,18}$ are depicted in Fig. 2(b), where it can also be seen that the approximation followed here can account acceptably for the observed behavior. The low-temperature portion of these curves show dependences with temperature of $\kappa(T) \propto T^{1.7}$ where the temperature exponent shows small but significant dependences with the sample thermal treatment. The relevant parameters are in this case $1.67 \times 10^{22} \mathrm{~cm}^{-3}$ for the normalization constant and $2 \times 10^{39} \mathrm{~J}^{-1} \mathrm{~cm}^{-3}$ for the TLS density of states, which obviously coincides with the value used in a recent analysis of the same material. ${ }^{2}$ Both cases corresponding to quenched and annealedunder-irradiation glasses at low temperature ${ }^{18}$ can also be reproduced with parameters not far apart from those of the previous data set, although the absence of measurements above $2 \mathrm{~K}$ hinders the reliable estimation of the required constants. The corresponding values for these quantities are $1.82 \times 10^{22} \mathrm{~cm}^{-3}$ and $3.6 \times 10^{39} \mathrm{~J}^{-1} \mathrm{~cm}^{-3}$ for the quenched glass and $4.3 \times 10^{22} \mathrm{~cm}^{-3}$ and $1.14 \times 10^{39}$ $\mathrm{J}^{-1} \mathrm{~cm}^{-3}$ for the annealed sample. Again, an increase of the ultrasonic ${ }^{11}$ sound velocity of $12 \%$ was required to fit the latter sample, which shows a somewhat smaller specific heat in the region of about $5 \mathrm{~K}$ than the quenched sample, with a maximum of $C(T) / T^{3}$ slightly displaced towards higher temperatures. It should be noted that the values corresponding to the first plateau region (1$10 \mathrm{~K})$ are rather close in both intermediate- and lowtemperature measurements.

As a comparison, Fig. 2(c) displays the experimental and calculated conductivities for a crystalline sample. Again, the low-temperature region of the experimental curve shows a significant deviation from a cubic temperature dependence, giving an exponent of $\approx 2.25$, and the location of the maximum of the curve at about $20 \mathrm{~K}$ is close to that of the annealed sample at high temperature [Fig. 2(a)]. The optimized values for the relevant parameters are $13.07 \mu \mathrm{m}$ for the average crystallite size, which can be compared to estimates of $5 \mu \mathrm{m}$ made by
Meissner et al. ${ }^{17}$ from an analysis of the conductivity by means of a Debye approach; a relatively large Rayleigh coefficient giving $A_{\text {Rayl }}=6.46 \times 10^{-41} \mathrm{~s}^{3}$, and a value for the anharmonic coefficient of $B=5.11 \times 10^{-24} \mathrm{~s}^{2} \mathrm{~K}^{-3}$. Although for temperatures above $100 \mathrm{~K}$ the simplified model used for the crystal should be supplemented by higher-order anharmonic terms to reproduce the conductivity in the full temperature range, it seems clear that a simple calculational scheme such as the one followed here can give an acceptable account of this transport property provided that a full frequency distribution instead of a Debye model is used for the calculation. In this respect, it is worth remarking that once the Debye density of states is replaced in the calculations by the true $Z_{\text {cryst }}(\omega)$ the resulting thermal conductivity will soon deviate from continuum (i.e., $T^{3}$ ) behavior. As a matter of fact, inspection of Fig. 1(c) shows that the quadratic frequency dependence of the frequency distribution is lost for frequencies higher than $0.8 \mathrm{THz}(\approx 38 \mathrm{~K})$, not far from the maximum of the thermal conductivity.

Finally, the frequency dependence of the mean free paths for a temperature about the first plateau are shown in Fig. 3. The most relevant features regarding the graph are (a) a strong drop at frequencies higher than $1 \mathrm{THz}$ is manifested by the contributions different from the librational one, whereas the latter one (and therefore the total path) shows a more smooth decrease with frequency; (b) the Rayleigh term, which is responsible for the alluded drop, becomes dominant at high frequencies, especially in the region corresponding to the gap of the frequency distribution as well as above the bond stretching peak (ca. from $8 \mathrm{THz}$ onwards); and (c) at the lowest frequencies sampled by the simulation, the phonon mean free path is about $100 \AA$, whereas at frequencies comparable with the temperature of the second plateau region $(\approx 1.5$ $\mathrm{THz}$ ) it drops to $\approx 10 \AA$. A subsequent increase up to $6 \mathrm{THz}(287 \mathrm{~K})$, is the responsible, in part, for the increase in $\kappa(T)$. The present findings thus contrast with those derived from simplified treatments based upon the

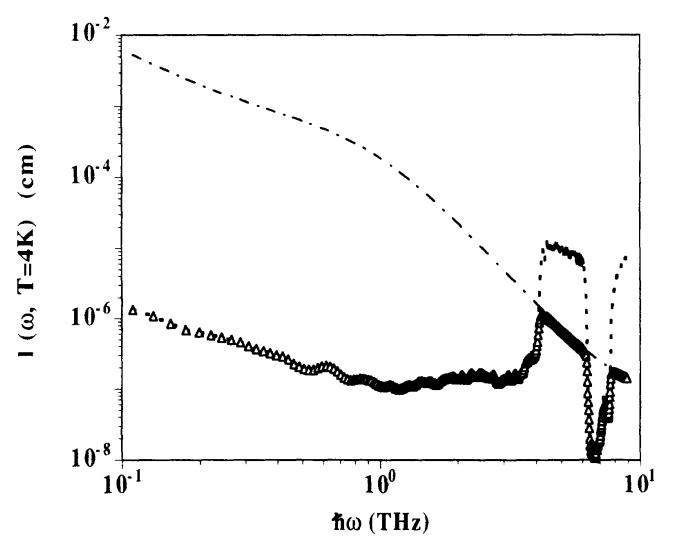

FIG. 3. Frequency dependence of the phonon mean free paths for $T=4 \mathrm{~K}$. The dash-dotted line gives the path corresponding to the sum of the (inverse) paths arising from Rayleigh, resonant, and relaxational scattering (see text). The dotted line shows $l_{\text {libr }}(\omega, T)$ and the open triangles depict the total path. 
dominant-phonon approximation, where the frequency dependence of the paths are derived directly from the thermal conductivity assuming a pure Debye behavior for the phonon density of states. Such inadequacy was previously remarked in semimicroscopic treatments for orientational glasses, ${ }^{4}$ and it is corroborated here for this structural glass.

The effects of sample annealing on the thermal properties of glasses have only recently been considered in detail. From pioneering work on metallic glasses and other amorphous materials, ${ }^{19}$ it has been evidenced that both a strong increase in the acoustic absorption (i.e., a concurrent decrease in thermal conductivity) and a reduction in the TLS density of states, alongside with a sizable increase in the velocity of sound, seem to be wellestablished consequences of the thermal treatment. From the procedure followed in this work, such effects can be put on a quantitative basis, without having to recourse to additional mechanisms (i.e., soft $\operatorname{modes}^{1,2}$ ) which are difficult to contrast with experimental measurements.

\section{DISCUSSION AND CONCLUSIONS}

The temperature dependence of the thermal conductivity of structural glasses can be analyzed following the steps delineated in this contribution. In the case herein considered, from the plateau region $(\approx 4 \mathrm{~K})$ onwards, the temperature dependence of $\kappa(T)$ can be accounted in full by the present approach, with some adjustment of two scale factors giving the density of librators and the Debye velocity of sound, in order to cope with the wide variations observed upon thermal treatment. Such a finding goes alongside with the fact that the bump in the $C_{v}(T) / T^{3}$ curves can also be reproduced by simulational means. At these temperatures, the low-energy librational excitations of a selenium chain (bond-angle torsions which are strongly coupled to the other degrees of freedom of the chain) constitute an effective mechanism for phonon scattering thus limiting the heat transport carried by long-wavelength phonons. The analysis of the low-temperature portion of thermal conductivity curves requires the introduction of additional scattering mechanisms, something which can be done within the standard TLS framework, and requires the use of an additional parameter giving the density of TLS states. From data presented here as well as in previous works, ${ }^{8}$ a clear connection between the anomalies in the specific heat at intermediate temperatures, the plateau in $\kappa(T)$, and a characteristic low-frequency peak (the boson peak) in the generalized susceptibility can be established. It is worth emphasizing at this point that the observed lowfrequency peak in coherent inelastic neutron scattering evidences a significant dependence with wave vector, ${ }^{20,21}$ which unveils the strong collective character of the excitations sampled at these length-scales. Such a fact constitutes an argument in support of those approaches postulating the existence of strongly interacting "defects," 4,3 in opposition to the standard TLS formulations, due to their inherent single-particle character.

\section{ACKNOWLEDGMENTS}

This work has been supported by DGICYT Grant No. PB92-0114-C03. Dr. R. Calemczuk from the CEN Grenoble (France) is kindly acknowledged for making available to us a copy of his Ph.D. dissertation.
${ }^{1}$ For a general description of these kind of models see Yu. M. Galperin, V.G. Karpov, and V.I. Kozub, Adv. Phys. 38, 669 (1989)

${ }^{2}$ U. Buchenau, Yu M. Galperin, V.L. Gurevich, D.A. Parshin, M.A. Ramos, and H.R. Schober, Phys. Rev. B 46, 2798 (1992).

${ }^{3}$ For a general appraisal see A.J. Leggett, Physica B 169, 322 (1991); see also C.C. Yu and A.J. Leggett, Comments Condens. Matter Phys. 14, 231 (1988); C.C. Yu, Phys. Rev. Lett. 63, 1160 (1989).

${ }^{4}$ J.P. Sethna, E.R. Grannan, and M. Randeria, Physica B 169, 316 (1991), and references therein.

${ }^{5}$ W.A. Phillips, Rep. Prog. Phys. 50, 1657 (1987).

${ }^{6}$ Calculations of the thermal conductivity from the thermal current correlation function are given in R.M. Ernst, S.R. Nagel, and G.S. Grest, Phys. Rev. B 43, 8070 (1991); and calculations regarding energy-fluctuations are given in D. Thirumalai and R.D. Mountain, ibid. 47, 479 (1993), and references therein. A recent appraisal of computer simulation methods for these purposes can be found in F. Yonezawa, in Solid State Physics, edited by H. Ehrenreich, and D. Turnbull (Academic, New York, 1991), Vol. 45, p. 179.

${ }^{7}$ U. Buchenau, M. Prager, N. Nücker, A.J. Dianoux, N. Ahmad, and W.A. Phillips, Phys. Rev. B 34, 5665 (1986).

${ }^{8}$ M. Garcia-Hernandez, F.J. Bermejo, B. Fåk, J.L. Mar- tinez, N.G. Almarza, and E. Enciso, Phys. Lett. A 175, 217 (1993); Phys. Rev. B 48, 149 (1993). Details about the employed potential functions and their validation are given in N.G. Almarza, E. Enciso, and F.J. Bermejo, Europhys. Lett. 17, 595 (1992).

${ }^{9}$ F.J. Bermejo, J. Alonso, A. Criado, F.J. Mompean, J.L. Martinez, and M. Garcia-Hernandez, Phys. Rev. B 46, 6173 (1992).

${ }^{10} \mathrm{An}$ account regarding this topic is given in F.J. Bermejo, M. Garcia-Hernandez, E. Enciso, J.L. Martinez, B. Fåk, and T. Mason, J. Noncryst. Solids (to be published).

${ }^{11}$ G. Carini, M. Cutroni, G. Galli, and F. Wanderlingh, J. Non-Cryst. Solids, 30, 61 (1978). Low-temperature data are given in G. Bellessa, C. Lemercier, and D. Caldemaison, Phys. Lett. 62A, 127 (1977). For the crystalline solid the temperature dependence of the orientationally averaged velocity of sound was calculated from the temperature dependence of the elastic constants given by M. Meissner and J. Mimkes, in The Physics of Selenium and Tellurium, edited by E. Gerlach and P. Grosse, Springer Series in Solid State Sciences Vol. 13 (Springer, Berlin, 1979), p. 74.

${ }^{12}$ J. Jäckle, L. Piche, W. Arnold, and S. Hunklinger, J. NonCryst. Solids 20, 365 (1976); D.P. Jones and W.A. Phillips, Phys. Rev. B 27, 3891 (1983).

${ }^{13}$ G.P. Srivastava, The Physics of Phonons (Adam Hilger, Bristol, 1990), Chaps. 6 and 7; see also, J. Phys. Chem. 
Solids 41, 357 (1980).

${ }^{14}$ R.C. Zeller and R.O. Pohl, Phys. Rev. B 4, 2029 (1971).

${ }^{15}$ P. Brüesch, Phonons: Theory and Experiments III, Phenomena Related to Phonons, Springer Series in Solid State Sciences Vol. 66 (Springer, Berlin, 1987), p. 76.

${ }^{16}$ N.G. Almarza, E. Enciso, and F.J. Bermejo, J. Chem. Phys. 99, 6798 (1993).

${ }^{17}$ M. Meissner and D. Wobig, in The Physics of Selenium and Tellurium, edited by E. Gerlach and P. Grosse, Springer Series in Solid State Sciences Vol. 13 (Springer, Berlin, 1979), p. 68, and references therein.

${ }^{18}$ R. Calemczuk, Ph.D. dissertation, Universite de Grenoble,
1983.

${ }^{19} \mathrm{~S}$. Hunklinger, in Heidelberg Colloqium on Glassy Dynamics, edited by J.L. van Hemmen and I. Morgenstern, Springer Lecture Notes in Physics Vol. 275 (Springer, Berlin, 1986), p. 94; also, H. Härdle, G. Weiss, S. Hunklinger, and F. Baumann, Z. Phys. B 65, 291 (1987).

${ }^{20}$ F.J. Bermejo, J.L. Martinez, M. Garcia-Hernandez, D. Martin, J. Alonso, and W.S. Howells, Europhys. Lett. 15, 509 (1991).

${ }^{21}$ J.B. Suck, H. Rudin, H.L. Gunterodt, and H. Beck, Phys. Rev. Lett. 50, 49 (1983). 